\title{
Propiedades psicométricas de la Escala de Actitudes sobre el Amor (LAS) en universitarios peruanos
}

\author{
Paula C. Lascurain Wais, María Claudia Lavandera Liria y Eduardo Manzanares Medina* \\ Universidad Peruana de Ciencias Aplicadas, Facultad de Psicología
}

Recibido, noviembre 4/2016

Concepto de evaluación, febrero 3/2017

Aceptado, mayo 3/2017
Referencia: Lascurain Wais, P.C., Lavandera Liria, M.C., Manzanares Medina, E. (2017). Propiedades psicométricas de la escala de actitudes sobre el amor en universitarios peruanos. Acta colombiana de Psicología, 20(2), 270-281. doi: http://www.dx.doi.org/10.14718/ACP.2017.20.2.13

Resumen

El objetivo de este estudio fue analizar la validez de constructo y la fiabilidad de la Escala de Actitudes sobre el Amor (Love Attitudes Scale [LAS]) de Hendrick \& Hendrick (1986). La muestra estuvo compuesta por 381 estudiantes universitarios de Lima, Perú, (61.7 \% mujeres) de entre 17 y 25 años de edad. Aparte del $L A S$, se aplicó el I-PANAS-SF adaptado por Gargurevich (2010), el cual evalúa el afecto positivo y negativo. Como resultados, el análisis factorial exploratorio del $L A S$ arrojó una solución de seis factores (Eros, Ludus, Storge, Pragma, Manía y Ágape) que explicaron el 47.87 \% de la varianza total. Las correlaciones entre las puntuaciones de los estilos Eros y Pragma y el afecto positivo fueron positivas y significativas; mientras que los estilos Manía y Ludus tuvieron correlaciones positivas y significativas con el afecto negativo. Utilizando el método de consistencia interna, la fiabilidad de los seis factores encontrados fue de .62 a .85; mientras que con el método testretest, los coeficientes de estabilidad fueron de .44 a .77. Estos resultados permiten concluir que el $L A S$ y sus puntuaciones son una medida válida y fiable para medir las actitudes hacia el amor en la población de universitarios de Lima, Perú.

Palabras clave: Actitudes hacia el amor, validez, confiabilidad, universitarios.

\section{Psychometric properties of the love attitudes scale in peruvian undergraduate students}

\begin{abstract}
The aim of this article was to analyze the construct validity and reliability of the Love Attitude Scale (LAS, Hendrick \& Hendrick, 1986). The sample consisted of 381 undergraduate students (61.7\% of them women) between the ages of 17 and 25 years old. The LAS and the I- PANAS - SF (adapted by Gargurevich, 2010) that evaluates positive and negative affect were applied. The exploratory factor analysis resulted in six factors (Eros, Ludus, Storge, Pragma, Mania and Ágape), that explained $47.87 \%$ of the total variance. The correlations between the score of Eros and Pragma styles and positive affect were positive and significant; while Manía and Ludus styles had positive and significant relationships with negative affect. Reliability by the method of internal consistency of the six factors found was from .62 to .85 ; while the stability coefficients for test- retest method were .44 to .77 . These results allow concluding that the LAS and their scores are valid and reliable to measure attitudes toward love in the college population of Lima.

Key words: Attitudes toward love, validity, reliability, university
\end{abstract}

* Av. Prolongación Primavera 2390 Monterrico - Santiago de Surco, Lima 33, Perú, Tel: +511 3133333 anexo 1140 , eduardo.manzanares@upc.pe 


\title{
Propriedades psicométricas da Escala de Atitudes sobre o Amor (LAS) em universitários peruanos
}

\begin{abstract}
Resumo
O objetivo deste estudo foi analisar a validade de construto e a confiabilidade da Escala de Atitudes sobre o Amor (Love Attitudes Scale [LAS]) de Hendrick e Hendrick (1986). A amostra foi composta por 381 estudantes universitários de Lima, Peru (61.7 \% mulheres) de 17 a 25 anos. Além da LAS, foi aplicado o I-PANAS-SF adaptado por Gargurevich (2010), o qual avalia efeito positivo e negativo. Como resultados, a análise fatorial exploratória da LAS mostrou uma solução de seis fatores (eros, agape, ludus, storge, mania e pragma) que explicaram 47.87 \% da variação total. As correlações entre as pontuações dos estilos eros e pragma e o afeto positivo foram positivas e significativas; enquanto os estilos mania e ludus tiveram correlações positivas e significativas com o afeto negativo. Utilizando o método de consistência interna, a confiabilidade dos seis fatores encontrados foi de .62 a .85; enquanto, com o método teste-reteste, os coeficientes de estabilidade foram de .44 a .77. Esses resultados permitem concluir que a LAS e suas pontuações são uma medida válida e confiável para medir as atitudes sobre o amor na população de universitários de Lima.

Palavras-chave: Atitudes sobre o amor, confiabilidade, universitários, validade.
\end{abstract}

\section{INTRODUCCIÓN}

El ser humano siempre se ha interesado por el tema de las relaciones interpersonales, la búsqueda de una pareja $y$, en especial, por el amor, que es considerado como un aspecto importante de la experiencia humana (Graham \& Christiansen, 2009). En los inicios de la historia de la cultura, el estudio del constructo del amor ha recibido una considerable atención y discusión desde la filosofía; por ejemplo, para Platón el amor es concebido como una emoción que existe de forma jerárquica, mientras que para Sócrates es una combinación de varios elementos (Wan, Hoesni, \& Chong, 2012). Sin embargo, desde la psicología científica no se había demostrado un interés por investigar acerca del amor, sino hasta las últimas tres décadas, donde se revisó e investigó de manera creciente este constructo (Todosijević, Arančić, \& Ljubinković, 2009).

Producto de este interés, surgieron como resultado diversas operacionalizaciones del constructo del amor, sobre todo con la medición de las actitudes, los estilos y las dimensiones del amor, como ocurre en los trabajos de Rubin en 1970, Bardis en 1978, Hatfield en 1986, o de Sternberg, con su Teoría Triangular del Amor (1986, 1987, 1997; citados por Carreño \& Serrano, 1995).

Una de las propuestas más importantes con respecto a los estilos de amor es la tipología elaborada por Lee en 1973 (Camacho et al., 2012; García et al., 2012; Ubillos, Zubieta, Páez, Deschamps, Ezeiza, \& Vera, 2002), ya que recoge los aportes de Platón, específicamente en su obra "El Banquete" — del año 380 A. C.—, donde propone tres formas de amor: el Eros, el Storge y el Ludus (García et al., 2012). A partir de esta tipología, Lee corrobora de manera empírica los estilos de amor basados en la teoría platónica, y le agrega tres estilos secundarios que surgen de la mezcla de los tres originales - la Manía, el Pragma y el Ágape-, para un total de seis actitudes o estilos de amor (Ubillos, Páez, \& Zubieta, 2004). Este autor representa los estilos de amor como aspectos de dominio cognitivo ligados al sistema de creencias y actitudes de cada individuo sobre las situaciones románticas de su vida (Hendrick \& Hendrick, 1986; Hendrick, Hendrick, \& Dicke, 1998), los cuales sirven para dirigir su comportamiento y experiencias hacia la persona que aman (Wan et al., 2012). Cada uno de estos seis estilos, aunque pueden tener cierto grado de relación, tienen características cualitativas diferenciadas, lo que hace a cada estilo independiente de todos los demás (Lee, 1973).

Continuando con esta teoría, Hendrick y Hendrick (1986) estructuraron un instrumento de medición de los estilos de amor denominado Love Attitudes Scale — LAS-, o Escala de Actitudes sobre el Amor, conformado por 42 ítems, que permite medir dichos estilos según la división entre: Eros, descrito como un amor pasional o romántico; Storge, entendido como el amor amistoso basado en la intimidad y el cariño; y Ludus, que sería un amor lúdico, manipulador y de poca implicación emocional. Adicionalmente, a partir de la mezcla o combinación de estos tres estilos, surgen otros tres tipos de amor: la Manía, entendida como un amor obsesivo y posesivo que surge de la combinación de Eros y Ludus; el Pragma, que lleva a la búsqueda lógica y racional de la pareja compatible ideal, y que se establece a partir de la mezcla entre Ludus y Storge; y el Ágape, entendido como un amor altruista de entrega total a la pareja, y que se constituye por la mezcla de Eros y Storge, (García et.al., 2012).

En resumen, Hendrick y Hendrick (1986) afirman que esta escala evalúa las actitudes hacia las relaciones amorosas a partir de un abordaje individual; y que, tomando en cuenta los seis estilos de amor como variables diferentes bajo una 
escala que describe cada forma de amar, es posible establecer a cada persona un perfil de su actitud hacia el amor.

Por otra parte, a pesar de haber teorizaciones distintas con respecto al amor, aparte del $L A S$ no se han desarrollado muchos instrumentos válidos para su medición (Brenlla, Brizzio, \& Carreras, 2004), exceptuando el Loving and Liking, de Rubin (1970), el Passionate Love Scale (PLS), de Hatfield y Sprecher (1986), y la Escala Triangular del Amor de Sternberg (1986; citado por Graham, 2011). Teniendo en cuenta que la investigación ha vinculado los altos niveles de amor romántico experimentado con una mayor satisfacción y estabilidad de la relación de pareja, además de mayores niveles de salud psicológica y general (Fehr, 2001; Graham 2011; Graham \& Christiansen, 2009), es notable la necesidad de contar con medidas válidas para tener una aproximación al constructo del amor y sus aspectos relacionados.

Adicionalmente, se han reportado pocos estudios de habla hispana que recojan evidencias de las propiedades psicométricas de instrumentos construidos para ese fin. Y, dentro estos, los que reportan resultados psicométricos sobre el $L A S$ son los realizados por Brenlla et al. (2004), Cooper y Pinto (2008), Otazzi (2009) y Rodríguez, Montgomery, Peláez y Salas (2003), que se describen a continuación.

Rodríguez et al. (2003) hicieron una investigación sobre de las actitudes amorosas y experiencias en el cortejo en adultos jóvenes de tres distintas culturas, utilizaron una muestra de 268 universitarios españoles, mexicanos y americanos de origen latino con edades entre los $17 \mathrm{y}$ 27 años, y usaron como instrumento el $L A S$-traducido y adaptado como Escala de Actitudes Amorosas (EEA)Con respecto a esta adaptación, los autores reportaron un análisis de confiabilidad en el que se observaron coeficientes alfa que iban desde .54 - para Storge — hasta .79 — para Pragma -; y reportaron como evidencias de validez de la estructura interna los resultados de un análisis de componentes principales (ACP), donde se encontró una solución de seis componentes similar a la del modelo original, aunque seis ítems contaron con problemas como: presentar cargas compartidas (el ítem 7 de Eros), pertenecer a otro componente distinto al teórico (el ítem 8 de Ludus y los ítems 15 y 19 de Storge) o tener una carga inferior a 30 (items 16 y 17 de Storge).

Si bien es cierto que en dicha investigación se realiza una primera exploración de las propiedades psicométricas del $L A S$, a nivel metodológico pueden observarse algunas limitaciones, tales como el uso del Little Jiffy (ACP, criterio de autovalores mayores a 1 y rotación varimax) como parte del análisis, estrategia poco recomendada cuando se pretende examinar de forma exploratoria la estructura interna de un instrumento (Dominguez-Lara \& Merino-Soto,
2016; Lloret-Segura, Ferreres-Traver, Hernández-Baeza, \& Tomás-Marco, 2014); o que en el reporte del coeficiente alfa no se aprecia el cálculo de sus intervalos de confianza, lo cual representa una práctica importante y recomendada actualmente debido a que este coeficiente puede estar influenciado por diversas características, como el número de ítems o el error muestral, y porque la confiabilidad de los puntajes no puede asumirse como estable en cada muestra evaluada (Dominguez-Lara \& Merino-Soto, 2015; SánchezMeca \& López-Pina, 2008).

Por otra parte, en el estudio de Brenlla et al. (2004) pueden encontrarse procedimientos de análisis psicométricos similares. En dicho estudio se aplicó el $L A S$ a una muestra de 280 adultos jóvenes argentinos con edad media de 27.4 años $(\mathrm{DE}=5.66)$, y se encontró, como parte de la estructura interna al aplicar ACP con rotación varimax, una solución factorial de seis componentes que en su conjunto explicaron el $41 \%$ de la varianza total; además, la distribución de los ítems fue similar a la original a excepción de cinco ítems que presentaron cargas factoriales bajas $(<.35)$-ítems 9 y 10 de Ludus, e ítems 15, 16 y 17 de Storge-, aunque se decidió mantenerlos. Por otra parte, la consistencia interna estimada a través del coeficiente alfa de Cronbach arrojó valores entre .40 para Ludus y .72 para Ágape.

Con respecto al estudio de Cooper y Pinto (2008), también se empleó como instrumento el $L A S$, el cual se aplicó a una muestra de 388 jóvenes universitarios bolivianos con edades entre los 18 y 24 años. Como parte de las propiedades psicométricas, los autores reportaron únicamente evidencias de confiabilidad por el método de consistencia interna, donde se obtuvieron coeficientes alfa de Cronbach entre .70 para Ludus y .89 para Ágape; sin embargo, no reportaron los intervalos de confianza ni alguna evidencia de validez para el $L A S$, como pudo haberse hecho con el análisis de la estructura interna.

Por otra parte, el estudio de Otazzi (2009), el único en el contexto peruano, aplica el $L A S$, con algunas adaptaciones lingüísticas en el fraseo de ciertos ítems, a una muestra de 71 adultos de ambos sexos con edades entre los 37 y 78 años. En este estudio se reportan evidencias de confiabilidad por el método de consistencia interna (coeficientes alfa entre .40 para Ludus y .88 para Eros), pero, de igual manera, se encuentra la ausencia de evidencias de validez. Asimismo, los resultados de confiabilidad se obtuvieron con muestras pequeñas - menores a 100 sujetos-.

Los estudios mencionados anteriormente provienen de investigaciones realizadas en muestras de habla hispana. Sin embargo, cabe mencionar la adaptación al portugués del $L A S$ realizada por De Andrade y García $(2009,2014)$, en la cual los autores evaluaron —en su segundo estudio - a una muestra brasileña conformada por 1530 adultos jóvenes de 
ambos sexos, de diversos estados de Brasil, y con una edad promedio de 25.5 años $(D E=8.7)$. Como parte del análisis factorial exploratorio con el método de extracción de ejes principales, y mediante el análisis paralelo, se determinó la extracción de seis factores que posteriormente fueron sometidos a una rotación oblicua (promax). Del análisis se obtuvo una versión de 37 ítems que distribuidos en los seis factores similares al modelo teórico y que explicaron el $45.3 \%$ de la varianza total. Dos ítems de Ludus, dos de Storge y uno de Manía fueron sacados de la versión final por presentar cargas factoriales bajas. Al hacer el análisis factorial confirmatorio, el modelo de seis factores (con 37 ítems) interrelacionados presentó mejores índices de ajuste $\left[\chi^{2}=1766.31, \mathrm{gl}=614(\mathrm{p}<.001), \chi^{2} / \mathrm{gl}=2.877\right.$, $\mathrm{RMR}=.066, \mathrm{GFI}=.88, \mathrm{AGFI}=.87 \mathrm{CFI}=.85$, RMSEA $(90 \% \mathrm{CI})=.50(.47-.52)]$. Asimismo, con el análisis de confiabilidad por medio del método de consistencia interna se encontraron coeficientes alfa de entre 67 para Storge y .84 para Ágape. Este estudio destaca por su representatividad y por la metodología empleada en el análisis de las propiedades psicométricas, a pesar de no corresponder a una versión en español este instrumento.

Como puede observarse, en los estudios señalados con muestras de habla hispana, Brenlla et al. (2004), Cooper y Pinto (2008), Otazzi (2009) y Rodríguez et al. (2003) abordaron el tema del amor empleando la escala de Hendrick y Hendrick (1986). Sin embargo, sus datos corresponden, en algunos casos, a muestras muy reducidas; y aunque se reportan evidencias de confiabilidad de las puntuaciones de la prueba, se aprecia que en la mayoría de estudios hay una ausencia de reportes de evidencia de validez, lo cual es un requisito necesario en todo proceso de validación y adaptación de test, y es altamente recomendado en de las directrices de la International Test Commission (Muñiz, Elosua, \& Hambleton, 2013).

Ahora bien, teniendo en cuenta lo revisado, a pesar de que el $L A S$ se ha empleado como parte de estudios relacionados al tema de la pareja, sobre todo evaluando satisfacción y compromiso, en el contexto peruano no existen evidencias psicométricas que avalen el uso de este instrumento en estudiantes universitarios (Otazzi, 2009), y las aproximaciones que hay no reportan las evidencias de validez y confiabilidad recomendadas. En ese sentido, debido a la necesidad de establecer una adaptación que cuente con rigor metodológico en cuanto al análisis de las evidencias validez y confiabilidad, el objetivo principal de la presente investigación es analizar las propiedades psicométricas del LAS en una muestra de universitarios de la ciudad de Lima, Perú.

En función del objetivo principal, a continuación se examinan las evidencias de confiabilidad (consistencia interna y estabilidad) y de validez (estructura interna y de relaciones con otras variables) de la escala en una muestra de estudiantes universitarios; de ahí que el presente estudio sea de tipo instrumental, ya que se incluye dentro de la categoría de trabajos que analizan las propiedades psicométricas de instrumentos psicológicos, específicamente en la adaptación de test ya existentes (Ato, López \& Benavente, 2013).

Por último, cabe mencionar la importancia de contar con escalas que permitan evaluar, con evidencias de validez y confiabilidad, los estilos de amor que asumen los estudiantes universitarios, ya que en este contexto se van consolidando las formas de relación social y afectiva con el otro, como ocurre en la amistad y en el amor; además, en este contexto, la elección de la pareja se presenta como una necesidad de afecto e interdependencia que comprende variables históricas, culturales, biológicas y psicosociales (Valdéz, González, \& Sánchez, 2005). Finalmente, como mencionan Rodríguez-Castro, Lameiras-Fernández, CarreraFernández y Vallejo-Medina (2013), en este proceso de socialización entran en juego la normas sociocultares del amor romántico, las cuales juegan un rol importante en los mitos o falsas creencias asociadas al amor. Hacer estudios al respecto permitirá comprender el alcance de aquellos aspectos emocionales y cognitivos que se asocian a la elección de una pareja, así como entender las actitudes de los estudiantes con respecto a las relaciones amorosas, y encontrar alternativas que contribuyan al fortalecimiento de sus relaciones y su bienestar (Galicia, Sánchez, \& Robles, 2013; Lema et al., 2009).

\section{MÉTODO}

\section{Participantes}

Para la obtención de la muestra se empleó un muestreo no probabilístico de tipo intencional; de este, se obtuvo un total de 393 estudiantes universitarios, de los cuales doce fueron descartados debido a que no contestaron de forma completa los instrumentos. De este modo, la muestra definitiva de 381 estudiantes estuvo compuesta por $61.7 \%$ de mujeres y $38.3 \%$ de varones, el rango de edad osciló entre los 17 y 25 años, y la edad promedio fue de 20.45 años $(D E=2.25)$; también, en cuanto a su situación sentimental, $62.2 \%$ no se encontraban en una relación, pero sí habían tenido alguna anteriormente, y $37.8 \%$ se encontraban en una relación. Del total de estudiantes, $54.3 \%$ fueron de Psicología y el resto pertenecían a carreras de negocios $(25.2 \%)$ e ingenierías $(20.5 \%)$. 


\section{Instrumentos}

Love Attitudes Scale (LAS, Hendrick \& Hendrick, 1986).

Se utilizó la traducción al español de Ubillos et al. (2004) denominada Escala de Actitudes sobre el Amor, la cual evalúa los seis estilos de amor propuestos por Lee (1973); estilos considerados como independientes, donde no se tiene en cuenta la distinción entre estilos primarios y secundarios (García et al., 2012). Esta escala se compone por 42 ítems clasificados en seis subescalas: Eros -ítems 1-7-, Ludus — ítems 8-14, Storge -ítems 15-21-, Pragma -ítems 22-28 - Manía —ítems 29-35-y Ágape -ítems 36-42-. En cada ítem, la escala de respuesta va del 1 (totalmente en desacuerdo) hasta el 5 (totalmente de acuerdo).

En tres estudios conducidos previamente se encontró que el coeficiente alfa promedio era de .80 (Hendrick \& Hendrick, 1989; Hendrick et al., 1998). Y, de acuerdo con el estudio de White (2003), se encontró que la consistencia de los coeficientes de los seis sub estilos de amor era de .71 para Eros, .75 para Ludus, .84 para Storge, .82 para Pragma, .71 para Manía y .84 para Ágape.

Escala de Afecto Positivo y Negativo - Forma Corta (I-PANAS-SF, Thompson, 2007). Consiste en la versión internacional reducida de la Escala de Afecto Positivo y Negativo (PANAS) de Watson, Clark y Tellegen (1988). Consta de 10 ítems, cinco por cada afecto, cuyas propiedades psicométricas han sido estudiadas en varios países, incluyendo Perú (Gargurevich, 2010), donde se realizó un análisis factorial confirmatorio (AFC) para corroborar la estructura factorial de la escala. Los índices de bondad de ajuste evaluados en el AFC para el modelo de dos factores resultaron bastante satisfactorios: $\mathrm{SB} \chi 2 / \mathrm{gl}=3.27(\mathrm{~S}-\mathrm{B} \chi 2$ $=111.19, \mathrm{gl}=34), \mathrm{RMSEA}=.084, \mathrm{GFI}=.92$. Además, las cargas factoriales de los ítems alcanzaron valores entre .27 y .83 , siendo todas significativas; y la correlación entre las escalas de afecto positivo y negativo fue negativa $\mathrm{y}$ significativa $(\mathrm{r}=-.52, p<.001)$ (Gargurevich, 2010). En dicho estudio, para ambos tipo de afecto se obtuvieron coeficientes alfa de Cronbach iguales a .72.

\section{Procedimiento}

En primer lugar, se tramitó el permiso necesario para acceder a las instituciones con el fin de evaluar a la muestra específica. Previo a la aplicación, se realizó un piloto con el fin de revisar aspectos lingüísticos de la versión empleada, el cual, además, se sometió a una revisión por jueces docentes universitarios y psicólogos que contaban experiencia en la investigación y grado mínimo de maestría-, quienes validaron las modificaciones realizadas a partir de la aplicación piloto.

De este procedimiento se determinó la no consideración del ítem 29 (Cuando algo no va bien con mi pareja, se me hace un nudo en el estómago) por considerarse poco pertinente para medir el estilo Manía, ya que al hacer el análisis del grado de acuerdo con respecto a su pertinencia, este resultó muy bajo $\left(\mathrm{V}_{\text {Aiken }}<.50\right)$. Luego del piloto, el proceso de aplicación se realizó en las aulas de las universidades; acá, se informó a los estudiantes que su participación en el estudio sería voluntaria y anónima, para lo cual se les hizo entrega de un consentimiento informado. La investigación contó con la aprobación de un Comité Evaluador de la Facultad de Psicología de la universidad donde se realizó la investigación.

\section{Análisis de datos}

Para el análisis de datos se da cuenta de evidencias de validez relacionadas con la estructura interna y con criterios externos; así como evidencias de confiabilidad, como el test-retest y la consistencia interna para el LAS. En cuanto a la evidencia de validez de constructo (análisis de la estructura interna), se identificó el número y composición de factores necesarios para resumir las puntuaciones en un conjunto grande de variables observadas. Para este fin, se aplicó un análisis factorial exploratorio (AFE), empleando el método de extracción de ejes principales con rotación promax, el cual resulta ser una opción frente a la violación del supuesto de normalidad multivariada (Lloret-Segura et al., 2014, Osborne, 2014).

Asimismo, se empleó el análisis paralelo para determinar el número de factores a extraer, método de simulación donde se comparan autovalores empíricos versus autovalores producidos al azar (Timmerman \& Lorenzo-Seva, 2011). Finalmente, se decide emplear una aproximación exploratoria en la medida que resulta ser menos restrictiva que un enfoque confirmatorio, y al aplicarla es posible obtener una primera aproximación a la estructura factorial del $L A S$ en el contexto peruano sin restricciones en las cargas de los ítems, ya que pueden identificarse aquellos ítems que tengan cargas cruzadas en determinadas dimensiones (Lloret-Segura et al., 2014).

Con el fin de complementar el presente estudio, se reportan evidencias de validez externa mediante el análisis de la relación entre el $L A S$ y el I-PANAS-SF, el cual fue seleccionado por la relación que guarda con el tema del amor y las relaciones de pareja, y debido a la existencia de investigaciones previas donde se emplearon ambas escalas (Neto, 2012). 
Con respecto a las evidencias de confiabilidad, específicamente test-retest (coeficiente de correlación intraclase, ICC), se aplicaron dos mediciones a una submuestra de 50 universitarios, esto con el fin analizar la estabilidad de los resultados en un intervalo de un mes entre ambas aplicaciones. En este caso, se consideró el criterio de Cicchetti (1994) para determinar los niveles de los coeficientes obtenidos: $<.40=$ pobre; $\geq .40=$ aceptable; $\geq .60=$ bueno; $\geq .75=$ excelente. Finalmente, en cuanto a la consistencia interna, se calculó la confiabilidad para los factores a través del coeficiente de consistencia interna alfa de Cronbach; y para su interpretación se tomó en cuenta el criterio de De Vellis (2012), donde se considera que coeficientes alfa a partir de .65 son considerados aceptables para fines de investigación, y los mayores a .80, como buenos. En ambos casos se reportan los respectivos intervalos de confianza al $95 \%$.

\section{RESULTADOS}

Inicialmente, se analizó la homogeneidad de los ítems mediante las correlaciones ítem-test corregidas $\left(r_{i t c}\right)$, para lo cual se retuvieron valores superiores a .20 (Kline, 1986); y en este procedimiento, se tuvo que eliminar el ítem 9 (Lo que mi pareja desconoce de mí, no debería llamarle la atención particularmente) por no cumplir dicho criterio $\left(r_{i t c}=.18\right)$. Además, se calcularon los estadísticos de tendencia central y de dispersión de los ítems (véase Tabla 1) en donde, a excepción del ítem 30, no se identificaron violaciones al supuesto de normalidad univariada, pues los índices de asimetría y curtosis fueron inferiores a $+/-1.5$ (Forero, Maydeu-Olivares, \& Gallardo-Pujol, 2009). Por esta razón, se empleó la matriz de correlaciones de Pearson. Adicionalmente, se empleó el análisis Mardia (1970) para la asimetría y curtosis multivariada, donde se halló un coeficiente de asimetría de 240.77, $g l=11480, p=1.0 \mathrm{y}$ un coeficiente de curtosis de $1801.94, p<.001$, por lo cual queda demostrada la ausencia de una distribución normal multivariada de los datos.

Previo a la aplicación del AFE, se halló una medida de adecuación muestral (KMO) de .81, considerado como aceptable (Ferrando \& Anguiano-Carrasco, 2010). Además, se obtuvo un puntaje estadísticamente significativo, $X^{2}(820)=4598.43, p<.001$ en el Test de Esfericidad de Bartlett, lo que significa que existe suficiente grado de relación entre los ítems del instrumento para poder analizarlos factorialmente.

Para extraer y determinar el número de factores se optó por realizar el análisis paralelo, en el cual se sugirieron seis factores que presentaban autovalores mayores que los generados por azar, lo cual estuvo acorde con el modelo original propuesto por Hendrick y Hendrick (1986). Al realizar el primer análisis, asumiendo los seis factores y empleando el método de ejes principales, se obtuvo que dichos factores explicaban el $46.37 \%$ de la varianza total explicada. Sin embargo, se identificó que el ítem 36 (Trato siempre de ayudar a mi pareja a sobrellevar los momentos dificiles) presentaba una carga factorial menor a $.20 \mathrm{y}$ se encontraba en un factor distinto al propuesto por el modelo teórico. Además, el ítem 17 (Espero permanecer para siempre junto a mi pareja) presentó cargas factoriales en tres factores que eran menores a .20. Dado que estos ítems no presentaron cargas aceptables, se optó por eliminarlos del análisis (Tabachnick \& Fidell, 2013).

Al realizar el segundo análisis, sin los ítems mencionados y aplicando una rotación Promax, se encontró que los seis factores extraídos explicaban el $47.87 \%$ del total de la varianza explicada. En la Tabla 1 se presentan los autovalores obtenidos, así como los respectivos porcentajes de varianza explicada que corresponden a los estilos Eros -ítems del 1 al 7-, Ágape -ítems del 37 al 42-, Pragma -ítems del 22 al 28-, Ludus -ítems 8 y del 10 al 14-, Manía -ítems del 30 al 35-, y Storge —ítems 15,16 y del 18 al 21-. En todos los casos, las cargas factoriales obtenidas fueron consideradas como aceptables, pues superan el valor mínimo recomendable por Tabachnick y Fidell (2013) de .30 .

Es importante resaltar que en el factor Pragma, los ítems 22 (Antes de comprometerse con una persona hay que considerar lo que éllella va a llegar a ser en la vida) y 23 (Hay que planificar cuidadosamente la vida antes de elegir pareja) presentaron carga compartida con el factor Storge. Finalmente, para Storge, el ítem 15 (Es difícil decir exactamente dónde termina la amistad y empieza el amor) presentó carga compartida con el factor Ludus (véase Tabla 1).

Asimismo, se obtuvieron las estimaciones de las comunalidades extraídas para los 38 ítems de la escala. Y, respecto a esto, es importante analizar las comunalidades, ya que los valores bajos $\left(h^{2}<.20\right.$, Child, 2006; $h^{2}<.30$; Costello \& Osborne, 2005) pueden generar una alteración significativa en los resultados del análisis factorial (Velicer \& Fava, 1998). En general, los resultados arrojaron comunalidades moderadas en la mayoría de los 38 ítems (media $\left.h^{2}=.40\right)$, y los valores más bajos fueron los de los ítems 8,13 y $16(.22, .22$ y .23 , respectivamente), mientras que los más altos fueron las comunalidades de los ítems 3 y 42 (.68 y .63 , respectivamente).

De igual manera, como también se aprecia en la Tabla 1, se obtuvieron coeficientes de fiabilidad de las puntuaciones que fueron considerados como aceptables para los estilos de 
Tabla 1

Estadísticos descriptivos, comunalidades, cargas factoriales y coeficientes de fiablidad de los items de la Escala de Actitudes sobre el Amor

\begin{tabular}{|c|c|c|c|c|c|c|c|c|c|c|c|}
\hline Ítem & Eros & Ágape & Pragma & Ludus & Manía & Storge & $\mathrm{h}^{2}$ & M & $D E$ & $\mathrm{~g}_{1}$ & $\mathrm{~g}_{2}$ \\
\hline 3. Amor intenso y satisfactorio. & .82 & & & & & & .68 & 3.76 & 1.03 & -.75 & .22 \\
\hline 4. Destinados el uno para el otro. & .74 & & & & & & .55 & 3.37 & 1.10 & -.34 & -.37 \\
\hline 2. Buena química entre la pareja. & .73 & & & & & & .55 & 4.11 & .96 & -1.11 & 1.06 \\
\hline 5. Rápida implicancia emocional. & .71 & & & & & & .53 & 3.63 & 1.05 & -.56 & -.26 \\
\hline 6. Comprensión mutua. & .64 & & & & & & .45 & 3.73 & 1.02 & -.62 & -.15 \\
\hline 7. Satisfacción del ideal de belleza física. & .47 & & & & & & .28 & 3.60 & 1.09 & -.64 & -.08 \\
\hline 1. Atracción física inmediata. & .45 & & & & & & .27 & 3.08 & 1.26 & -.18 & -.90 \\
\hline 42. Soportar todo por la pareja. & .39 & .75 & & & & & .63 & 3.45 & 1.18 & -.41 & -.61 \\
\hline 41. Amor incondicional. & & .74 & & & & & .55 & 3.42 & 1.25 & -.41 & -.79 \\
\hline 40. Estar a disposición de la pareja. & & .67 & & & & & .46 & 2.79 & 1.26 & .09 & -.99 \\
\hline 39. Sacrificio de los propios deseos. & & .68 & & & .42 & & .51 & 2.65 & 1.20 & .16 & -.83 \\
\hline 38. Su felicidad por encima de la mía. & & .67 & & & & & .51 & 3.30 & 1.23 & -.34 & -.77 \\
\hline 37. Prefiero sufrir yo antes que mi pareja. & & .65 & & & .37 & & .44 & 3.15 & 1.22 & -.12 & -.78 \\
\hline 25. Que mi familia acepte a mi pareja. & & & .71 & & & & .52 & 2.81 & 1.30 & .13 & -1.08 \\
\hline 26. Saber si será buen(a) padre/madre. & & & .70 & & & & .51 & 2.79 & 1.25 & .05 & -1.01 \\
\hline 27. Me ayudará en mi carrera profesional. & & & .67 & & & & .47 & 3.07 & 1.38 & -.11 & -1.22 \\
\hline 28. Verificar compatibilidad genética. & & & .49 & & & & .30 & 1.81 & 1.10 & 1.20 & .49 \\
\hline 24. Que mi pareja sea de mi medio social. & & & .46 & & & & .29 & 2.67 & 1.22 & .16 & -.97 \\
\hline 23. Planificar mi vida antes de elegir pareja. & & & .45 & & & .48 & .34 & 3.44 & 1.16 & -.31 & -.67 \\
\hline $\begin{array}{l}\text { 22. Considerar lo que mi pareja quiere ser en } \\
\text { la vida. }\end{array}$ & & & .43 & & & .46 & .36 & 3.46 & 1.10 & -.44 & -.30 \\
\hline 14. Gilear (cortejar) con diferentes personas. & & & & .60 & & & .38 & 2.10 & 1.26 & .78 & -.64 \\
\hline $\begin{array}{l}\text { 12. Que se entere que estuve con otras perso- } \\
\text { nas. }\end{array}$ & & & & .58 & & & .38 & 2.62 & 1.34 & .30 & -1.10 \\
\hline 10. Relaciones con dos personas a la vez. & & & & .55 & & & .34 & 1.73 & 1.20 & 1.45 & .79 \\
\hline $\begin{array}{l}\text { 11. Recuperarse rápido de una decepción } \\
\text { amorosa. }\end{array}$ & & & & .48 & & & .26 & 2.27 & 1.27 & .69 & -.55 \\
\hline $\begin{array}{l}\text { 8. Inseguridad de mi pareja sobre mi compro- } \\
\text { miso. }\end{array}$ & & & & .41 & & & .22 & 2.69 & 1.18 & .10 & -.86 \\
\hline 13. Distanciamiento debido a la dependencia. & & & & .37 & & & .22 & 3.08 & 1.22 & -.16 & -.88 \\
\hline 32. Ponerse mal por falta de atención. & & & & & .59 & & .44 & 3.29 & 1.15 & -.32 & -.65 \\
\hline 35. Hacer cosas sin sentido cuando me ignora. & & & & & .61 & & .40 & 2.54 & 1.27 & .37 & -.93 \\
\hline 33. Desconcentración al pensar en mi pareja. & & & & & .59 & & .37 & 2.70 & 1.14 & .19 & -.77 \\
\hline 31. No dormir por estar enamorado. & & & & & .58 & & .36 & 2.87 & 1.25 & .01 & -1.04 \\
\hline 34. Intranquilo al pensar que está con otro(a). & & .32 & & & .57 & & .36 & 2.96 & 1.28 & .01 & -1.01 \\
\hline 30. Pensar en suicidio, tras una ruptura. & & & & & .40 & & .27 & 1.59 & 1.03 & 1.66 & 1.74 \\
\hline 18. El amor profundo nace de una amistad. & & & & & & 67 & .46 & 3.29 & 1.29 & -.26 & -.92 \\
\hline 21. Relación satisfactoria viene de amistad. & & & & & & .59 & .35 & 3.37 & 1.32 & -.38 & -.97 \\
\hline 20. El amor es una amistad profunda. & & & & & & .50 & .26 & 3.40 & 1.25 & -.33 & -.87 \\
\hline 19. Nuestra amistad se convirtió en amor. & & & & & & .36 & .24 & 3.14 & 1.18 & -.13 & -.84 \\
\hline 16. No se puede amar sin afecto previo. & & & & & & .31 & .23 & 3.84 & 1.25 & -.83 & -.39 \\
\hline 15. Difícil discernir entre amistad y amor. & & & & .39 & & .30 & .28 & 2.91 & 1.28 & .09 & -.98 \\
\hline Autovalor & 4.94 & 3.93 & 3.43 & 2.26 & 2.02 & 1.62 & - & - & - & - & - \\
\hline$\%$ varianza explicada & $13.00 \%$ & $10.34 \%$ & $9.03 \%$ & $5.94 \%$ & $5.31 \%$ & $4.25 \%$ & - & - & - & - & - \\
\hline $\begin{array}{l}\alpha \\
{[\mathrm{IC}, 95 \%]}\end{array}$ & $\begin{array}{c}.83 \\
{[.80, .86]}\end{array}$ & $\begin{array}{c}.85 \\
{[.82, .88]}\end{array}$ & $\begin{array}{c}.77 \\
{[.73, .81]}\end{array}$ & $\begin{array}{c}.67 \\
{[.61, .72]}\end{array}$ & $\begin{array}{c}.73 \\
{[.68, .77]}\end{array}$ & $\begin{array}{c}.62 \\
{[.56, .68]}\end{array}$ & - & - & - & - & - \\
\hline $\begin{array}{l}\text { ICC } \\
{[\mathrm{IC}, 95 \%]}\end{array}$ & $\begin{array}{c}.77 \\
{[.60, .87]}\end{array}$ & $\begin{array}{c}.62 \\
{[.32, .78]}\end{array}$ & $\begin{array}{c}.57 \\
{[.22, .75]}\end{array}$ & $\begin{array}{c}.44 \\
{[.01, .68]}\end{array}$ & $\begin{array}{c}.67 \\
{[.41, .81]}\end{array}$ & $\begin{array}{c}.72 \\
{[.50, .84]}\end{array}$ & - & - & - & - & - \\
\hline
\end{tabular}

Nota. $h^{2}=$ comunalidad; $\mathrm{g} 1=$ asimetría; $\mathrm{g}_{2}=$ curtosis; $\mathrm{ICC}=$ coeficiente de correlación intraclase. 
amor analizados, excepto para los estilos Storge y Ludus, cuyo intervalo de confianza estuvo por debajo del mínimo aceptable $(\alpha<.65)$. Como parte de la confiabilidad de estabilidad y con el objetivo de medir el nivel de concordancia entre las dos medidas cuantitativas del $L A S$, se utilizó el método test-retest, el cual se calculó mediante el Coeficiente de Correlación Intraclase (ICC) y sus respectivos intervalos de confianza (IC) al $95 \%$. De este modo, se encontró que los estilos Eros, Manía y Storge presentaron buenos coeficientes test-retest (mayores a .60), mientras que para los estilos Ágape y Pragma fueron aceptables (mayores a .40) (Cicchetti, 1994; Prieto, Lamarca, \& Casado, 1998; Serra-Mayoral \& Peña-Casanova, 2006), y para el estilo Ludus fue bajo, ya que a pesar de ser igual a .44, el límite inferior del intervalo de confianza es cercano a cero.

Como parte de las evidencias de validez convergente y divergente, se procedió a correlacionar los seis estilos de amor con el afecto positivo y negativo del I-PANAS-SF (Gargurevich, 2010). Para tal fin, se empleó la prueba de correlación rho de Spearman, ya que en al análisis de normalidad, mediante la prueba de Kolmogorov-Smirnov, todas las distribuciones de las variables resultaron estadísticamente significativas $(p<.05)$, dado que no se aproximaron a una distribución normal. Tal como se observa en la Tabla 2, se encontraron correlaciones positivas y estadísticamente significativas entre el afecto positivo y los estilos de amor Eros $\left(r_{s}=.23, \mathrm{p}<.001, \mathrm{IC}[95 \%]=.13, .32\right)$ y $\operatorname{Pragma}\left(r_{s}=.17\right.$, $\mathrm{p}<.001, \operatorname{IC}[95 \%]=.07, .27)$. En cuanto al afecto negativo, se encontró una relación negativa y significativa con el estilo de amor $\operatorname{Eros}\left(r_{s}=-.14, p<.05\right.$, IC[95 \%] $\left.=-.23,-.04\right)$, $\mathrm{y}$, por el contrario, correlaciones positivas y significativas con los estilos de amor Ludus $\left(r_{s}=.15, p<.05\right.$, IC[95\%] $=.05, .25)$ y Manía $\left(r_{s}=.29, p<.001, \mathrm{IC}[95 \%]=.20\right.$, .38 ), y las correlaciones restantes no resultaron estadísticamente significativas. De estas relaciones obtenidas, en términos interpretativos y atendiendo a los límites de los intervalos de confianza, se consideran importantes la relación positiva entre Eros y el afecto positivo, así como la relación positiva entre Manía y el afecto negativo. En ambos casos, los intervalos se encontraron en un rango de tamaño del efecto entre bajo $(.10 \leq \mathrm{r}<.30)$ y moderado $(.30 \leq \mathrm{r}<.50)$ (Cohen, 1998).

En cuanto a las correlaciones entre los estilos de amor en el $L A S$, se encontraron correlaciones positivas y estadísticamente significativas entre Eros y Ágape $\left(r_{s}=.25, p<.001\right.$, $\operatorname{IC}[95 \%]=.15, .34) ;$ Ludus y Manía $\left(r_{s}=.24, p<.001\right.$, $\mathrm{IC}[95 \%]=.14, .33)$; Pragma y Storge $\left(r_{s}=.25, p<.001\right.$, IC $[95 \%]=.15, .34)$; y Ágape y Mania $\left(r_{s}=.35, p<.001\right.$, $\mathrm{IC}[95 \%]=.26, .44)$. Cabe mencionar que las correlaciones obtenidas estuvieron dentro de lo esperable en la medida que el afecto positivo se relacionó positivamente con los estilos de amor más adaptativos y que implican un mayor compromiso; mientras que el afecto negativo se correlacionó positivamente con los estilos más desadaptativos y de menor compromiso. Asimismo, se debe considerar que en cuanto a la magnitud de las correlaciones analizadas, estas fueron, en su mayoría, de valores bajos.

\section{DISCUSIÓN}

Para el presente trabajo se tuvo como objetivo el estudio de las propiedades psicométricas de la Escala de Actitudes sobre el Amor ( $L A S$, por sus siglas en inglés) de Hendrick y Hendrick (1986), en la versión española de Ubillos et al. (2004), en un grupo de universitarios de Lima, Perú. Los resultados encontrados demuestran propiedades psicométricas satisfactorias para el $L A S$ tanto en los criterios de evidencias de validez como de confiabilidad de las puntuaciones analizadas; lo cual avala su uso para efectos de investigaciones en muestras de universitarios peruanos.

En cuanto a la confiabilidad por consistencia interna de las puntuaciones, se encontraron coeficientes alfa entre buenos y aceptables (De Vellis, 2012) a excepción del estilo

Tabla 2

Matriz de correlaciones entre los estilos de amor y el afecto positivo y negativo ${ }^{a}$

\begin{tabular}{|c|c|c|c|c|c|c|c|c|c|c|}
\hline & $\mathrm{M}$ & $\mathrm{DE}$ & 1 & 2 & 3 & 4 & 5 & 6 & 7 & 8 \\
\hline 1. Afecto positivo. & 3.78 & 0.59 & - & & & & & & & \\
\hline 2. Afecto negativo. & 2.60 & 0.56 & $-.18 * *$ & - & & & & & & \\
\hline 3. Eros. & 3.61 & 0.76 & $.24 * *$ & $-.14 * *$ & - & & & & & \\
\hline 4. Ludus. & 2.41 & 0.76 & .04 & $.15^{* *}$ & $-.11 *$ & - & & & & \\
\hline 5. Storge. & 3.33 & 0.74 & .03 & $.12 *$ & -.05 & $.20 * *$ & - & & & \\
\hline 6. Pragma. & 2.86 & 0.79 & $.17 * *$ & -.02 & .01 & $.17 * *$ & $.25 * *$ & - & & \\
\hline 7. Manía. & 2.66 & 0.77 & -.09 & $.29 * *$ & .01 & $.24 * *$ & $.14 * *$ & $.14 * *$ & - & \\
\hline 8. Ágape. & 3.13 & 0.92 & -.01 & .05 & $.25^{* *}$ & -.01 & .08 & $-.12 *$ & $.35 * *$ & - \\
\hline
\end{tabular}

Nota. ${ }^{\text {a }}$ Las correlaciones presentadas corresponde al coeficiente de correlación de rho de Spearman. ${ }^{*} p<.05 ; * * p<.01$. 
Storge, que presentó una baja fiabilidad. Estos coeficientes resultaron ser más altos en comparación con los obtenidos por Rodríguez et al. (2003) y Brenlla et al. (2004); y similares a los obtenidos por Hendrick y Hendrick (1986), Otazzi (2009) y De Andrade y García (2014), aunque cabe mencionar que en dichos estudios no se reportaron los intervalos de confianza.

En los estudios mencionados, los estilos Ludus y Storge fueron los que presentaban los coeficientes más bajos, y esto coincide con el presente estudio, aunque aquí las magnitudes fueron más altas. Adicionalmente, la mínima variabilidad encontrada entre la fiabilidad de los puntajes puede deberse a la variabilidad de las muestras (Prieto \& Delgado, 2010) y al mantenimiento de algunos ítems con cargas factoriales bajas, como en el caso de los ítems 9 (Ludus) y 15 (Storge); y esto se corrobora con lo encontrado en el meta-análisis de Graham y Christiansen (2009), quienes sostienen que los resultados actuales que demuestran las diferencias en la fiabilidad de las puntuaciones de las medidas de amor son debidas a las características propias de las muestra. Por estas razones, es importante tomar en cuenta este aspecto de la fiabilidad para poder realizar interpretaciones sustantivas de las puntuaciones que se obtengan en diversas muestras.

Por otra parte, en cuanto a la estabilidad del instrumento por el método test-retest se observó que los coeficientes de correlación intraclase también fueron satisfactorios, a pesar de que en los estilos Ludus y Pragma se evidenció un indicador bajo que pudo darse por efecto de la aplicación luego de un mes, periodo en el cual algunos participantes cambiaron su situación sentimental - como se registró en la ficha sociodemográfica-; o por el tamaño de la muestra, que fue bastante pequeño. De ahí que en estos estilos los coeficientes hayan sido considerablemente inferiores a los hallados por Hendrick y Hendrick (1986), quienes hicieron el estudio con 112 estudiantes en un lapso de seis semanas. A pesar de esto, en líneas generales se aprecia que los puntajes obtenidos con el $L A S$ son consistentes y estables en el tiempo.

Con respecto a la validez relacionada a la estructura interna, los resultados obtenidos a través del Análisis Factorial Exploratorio corroboraron la estructura original de seis factores (Hendrick \& Hendrick, 1986), los cuales explican el $47.87 \%$ de la varianza con cargas factoriales altas, superiores casi en su totalidad a .30. Esto pone de manifiesto que los reactivos considerados contribuyen de manera significativa a la medición de los estilos de amor, y que brindan una solidez factorial (Zwick \& Velicer, 1986); sin embargo, para la presente investigación se tuvieron que eliminar los ítems 17 (Espero permanecer para siempre junto a mi pareja), 36 (Trato siempre de ayudar a mi pareja a sobrellevar los momentos difíciles) y 29 (Cuando algo no va bien con mi pareja, se me hace un nudo en el estómago).

La eliminación de estos ítems se debió, en el caso del 29, a un acuerdo entre los jueces respecto a la interpretación literal y figurativa del mismo; mientras que los ítem $36 \mathrm{y}$ 17 se eliminaron porque presentaban una carga factorial menor a 20 y porque presentaban cargas en tres factores, lo que estaría implicando que en la muestra evaluada dichos reactivos no estarían representando los estilos para los que teóricamente fueron elaborados. Con respecto a estas eliminaciones, el caso del ítem 17 coincide con los resultados de Brenlla et al. (2004), De Andrade y García (2014) y Rodríguez et al. (2003), por lo que la poca representatividad de ese ítem en su estilo teórico podría ser, incluso, un tema cultural.

También, se hallaron ítems con cargas en dos factores, como el 15 (Es dificil decir exactamente dónde termina la amistad y empieza el amor) en los estilos Storge (estilo teórico) y Ludus; y los ítems 22 (Antes de comprometerse con una persona hay que considerar lo que él/ella va a llegar a ser en la vida) y 23 (Hay que planificar cuidadosamente la vida antes de elegir pareja) en los estilos Pragma (estilo teórico) y Storge. El caso del ítem 15 coincide con lo encontrado en el análisis de Rodríguez et al. (2003). Con respecto a esto, y como explicación, se puede decir que diversas causas pueden ser planteadas para explicar que un ítem cargue en dos factores, entre ellas, y para los ítems mencionados, puede explicarse tomando como base la tipología de Lee (1973), pues el estilo Pragma se forma de la combinación de características de los estilos Ludus y Storge, y, por tanto, ciertas actitudes pueden ser compartidas y estar relacionadas. Esto se corrobora al analizar las correlaciones obtenidas entre dichos estilos (De Andrade \& García, 2014). En este sentido, por dichas razones se tomó la decisión, en estos casos particulares, de mantener los ítems a pesar de presentar cargas compartidas.

En líneas generales, pude decirse que en cuanto a las dimensiones de la Escala de Actitudes hacia el Amor de Hendrick y Hendrick (1986) se encuentra que la estructura de seis factores concuerda con la encontrada en los estudios de Rodríguez et al. (2003) en muestras de universitarios de habla hispana; De Andrade y García (2009) en una muestra brasileña; y Brenlla et al. (2004) en adultos jóvenes argentinos. Estos resultados indican que existe un buen ajuste del modelo teórico propuesto por Hendrick y Hendrick (1986) a los datos empíricos de la muestra peruana.

Además, estos resultados adicionan evidencia con respecto al grado de consistencia y estabilidad de la estructura interna del $L A S$ en distintas muestras, pues los ítems funcionan, en gran medida, de manera similar, lo cual apoya la generalización del modelo teórico del constructo 
a pesar de que este se trate de un estudio exploratorio que requiere de un método confirmatorio y replicaciones que aporten a las evidencias halladas en el mismo. También, se observó que el estilo Eros es el factor que mayor varianza retiene y el de mayor preferencia tanto en hombres como en mujeres; dicho resultado muestra de forma clara la vigencia del modelo del amor romántico en el medio peruano, específicamente en jóvenes universitarios, y esto coincide con resultados obtenidos en muestras de jóvenes españoles (Ferrer et al. 2008).

Por otra parte, en cuanto a las correlaciones entre los estilos de $L A S$, estas se analizaron con base en el criterio de su magnitud, y se obtuvo que los coeficientes de correlación fueron en su mayoría estadísticamente significativos, aunque de magnitud baja. Este aspecto corrobora el hecho de que, si bien es cierto que existe cierto grado de asociación entre las dimensiones (De Andrade \& García, 2014), cada estilo mantiene también independencia frente a los demás; y esto coincide con lo planteado por Hendrick \& Hendrick (1986).

Adicionalmente, lo hallado con respecto a las correlaciones con el afecto positivo y negativo refleja el hecho de que los afectos positivos están más relacionados con estilos que buscan el agrado y la simpatía con uno mismo y con los demás, mientras que los afectos negativos llevan a realizar evaluaciones negativas de desagrado con uno mismo y antipatía hacia los otros (Ubillos et al., 2004; Neto, 2012). De ahí que el estilo Eros, que implica el amor romántico, se asocie de forma positiva con el efecto positivo y de manera inversa con el negativo; $y$, por otra parte, que los estilos Ludus (amor de poca implicancia emocional) y Manía (amor obsesivo) presentaran relaciones positiva con el afecto negativo, ya que a la larga dichos estilos llegan a afectar el bienestar de la persona y de la relación. Esto resulta de gran importancia ya que el afecto puede influir en la atracción hacia la pareja (Jonason \& Kavanagh, 2010; Ubillos et al., 2004).

Cabe mencionar que al tratarse de una primera exploración de la estructura factorial del $L A S$, se planteó un diseño a nivel exploratorio y no se pudieron utilizar otros procedimientos como el Análisis Factorial Confirmatorio (AFC), esto porque se quería tener la menor restricción en cuanto a las cargas de los ítems. Lo que está alineado con las afirmaciones de que el AFC puede llegar a diferir en la confirmación de estructuras factoriales apoyadas en los análisis exploratorios previos debido a su carácter restrictivo (Ferrando \& Anguiano-Carrasco, 2010; LloretSegura et al., 2014).

Por estas razones, se recomienda que para futuras investigaciones se replique el modelo encontrado en el presente estudio, pero en una muestra más amplia (esto teniendo en cuenta que las comunalidades fueron moderadas y, en algunos casos, menores a .30), y que se utilicen métodos confirmatorios para analizar la estructura interna del $L A S$, además de que se analice la bondad de ajuste del modelo factorial encontrado y se revisen aspectos relacionados a la invarianza factorial. También, se recomienda utilizar una muestra más variada en lo que respecta a la carrera, pues haber contado con un porcentaje alto de estudiantes de psicología podría considerarse como un posible sesgo en las respuestas, dadas las características de dichos alumnos.

Por último, se concluye que los resultados hallados indican que el $L A S$ aplicado en estudiantes universitarios peruanos cuenta con adecuadas propiedades psicométricas que permiten considerarlo como una medida válida y confiable que puede ser empleada en investigaciones futuras como instrumento de medición de los estilos de amor.

\section{REFERENCIAS}

Ato, M., López, J. J., \& Benavente, A. (2013). Un sistema de clasificación de los diseños de investigación en psicología. Anales de Psicología, 29(3), 1038-1059. doi: http://dx.doi. org/10.6018/analesps.29.3.178511.

Brenlla, M. E., Brizzio, A., \& Carreras, A. (2004). Actitudes hacia el amor y apego. Psicodebate, 4, 7-23. doi: http:// dx.doi.org/10.18682/pd.v4i0.491.

Camacho, J. M., Regalado, P., Carrea, G., Grosso, C., Geleazzi, F., Gunther, G., ... Ramos, J. (2012). Actitudes hacia el amor y Estilos de Humor en mujeres y varones: ¿Nos diferencia el sexo o el género? Psiencia: Revista Latinoamericana de Ciencia Psicológica, 4(1), 13-27. Recuperado de http://www.psiencia.org/ojs/index.php/psiencia/article/ view/86.

Carreño, M. \& Serrano, G. (1995). Análisis de Instrumentos para la medida del amor. Revista de Psicología Social, 10(2), 131-148.

Child, D. (2006). The essentials of factor analysis. ( $3^{\text {rd }}$ ed.). New York, NY: Continuum International Publishing Group.

Cicchetti, D. V. (1994). Guidelines, criteria, and rules of thumb for evaluating normed and standardized assessment instruments in psychology. Psychological Assessment, 6(4), 284290. doi: http://dx.doi.org/10.1037/1040-3590.6.4.284.

Cohen, J. (1988). Statistical power analysis for the behavioral sciences $\left(2^{\text {nd }}\right.$ ed.). Hillsdale, NJ: Lawrence Erlbaum.

Cooper, V. \& Pinto, B. (2008). Actitudes ante el amor y la Teoría de Sternberg. Un estudio correlacional en jóvenes universitarios de 18 a 24 años de edad. AJAYU, 6(2), 181-206. Recuperado de http://www.scielo.org.bo/scielo. php?script=sci_arttext\&pid=S2077-21612008000200004.

Costello, A. B. \& Osborne, J. W. (2005). Best Practices in Exploratory Factor Analysis: Four Recommendations for Getting the Most From Your Analysis. Practical Assessment, 
Research \& Evaluation, 10(7). Recuperado de http://pareonline.net/pdf/v10n7.pdf.

De Andrade, A. L. \& García, A. (2009). Atitudes e crenças sobre o amor. Versão brasileira da Escala de Estilos de Amor. Interpersona: An International Journal on Personal Relationships, 3(1), 89-102. Recuperado de http://interpersona.psychopen.eu/article/download/34/pdf.

De Andrade, A. L. \& García, A. (2014). Escala de Crenças sobre Amor Romântico: Indicadores de Validade e Precisão. Psicologia: Teoria e Pesquisa, 30(1), 63-71. doi: http://dx.doi.org/10.1590/S0102-37722014000100008.

De Vellis, R. (2012). Scale development: Theory and applications ( $4^{\text {th }}$ ed.). Thousand Oakes, C.A: Sage.

Dominguez-Lara, S. \& Merino-Soto, C. (2015). [Carta al Editor] ¿Por qué es importante reportar los intervalos de confianza del coeficiente alfa de Cronbach? Revista Latinoamericana de Ciencias Sociales, Niñez y Juventud, 13(2), 13261328. Recuperado de http://revistaumanizales.cinde.org.co/ index.php/Revista-Latinoamericana/article/view/2030/629.

Dominguez-Lara, S. \& Merino-Soto, C. (2016). [Carta ao Editor] Sobre o uso do Little Jiffy na validação dos testes: comentários a Ávila e colaboradores. J Bras Psiquia$t r, 65(2)$, 196-197. doi: http://dx.doi.org/10.1590/00472085000000123.

Fehr, B. (2001). The status of theory and research on love and commitment. In G. J. O. Fletcher \& M. S. Clark (Eds.), Blackwell handbook of social psychology: Interpersonal processes (pp. 331-356). Oxford, UK: Blackwell.

Ferrando, P. J., \& Anguiano-Carrasco, C. (2010). El análisis factorial como técnica de investigación en psicología. $P a$ peles del Psicólogo, 31(1), 18-33. Recuperado de http:// www.papelesdelpsicologo.es/resumen?pii=1793.

Ferrer, V., Bosch, E., Navarro, C., Ramis, M. C., \& García, E. (2008). El concepto de amor en España. Psicothema, 20 (4), 589-595. Recuperado de http://www.psicothema.com/ psicothema. asp?id $=3527$.

Forero, C. G, Maydeu-Olivares, A., \& Gallardo-Pujol, D. (2009). Factor analysis with ordinal indicators: A monte Carlo study comparing DWLS and ULS estimation. Structural Equation Modeling, 16(4), 625-641. doi: http:// dx.doi.org/10.1080/10705510903203573.

Galicia, I., Sánchez, A., \& Robles, F. J. (2013). Relaciones entre estilos de amor y relaciones violentas en adolescentes. Psicología desde el Caribe, 30(2), 211-235. Recuperado de http://rcientificas.uninorte.edu.co/index.php/psicologia/ article/view/4854/6916.

García, M. E., Garnica, M. C., González, N., Márquez, M., Martín, M., Pérez, M. M., \& Vico, M. N. (2012). Las mujeres viven la relación romántica diferente al hombre. Revista Electrónica de Investigación Docencia Creativa, 1, 95-100. Recuperado de http://digibug.ugr.es/handle/10481/21967\#. WUIEVeuGNpg.
Gargurevich, R. (2010). Propiedades psicométricas de la versión internacional de la Escala de Afecto Positivo y Negativo- forma corta (I- SPANAS SF) en estudiantes universitarios. Persona, 13, 31-42. Recuperado de https://revistas.ulima.edu.pe/index.php/Persona/article/ download/263/250.

Graham, J. (2011). Measuring love in romantic relationships: A meta-analysis. Journal of Social and Personal Relationships, 28(6), 748-771. doi: http://dx.doi. org $/ 10.1177 / 0265407510389126$.

Graham, J. \& Christiansen, K. (2009). The reliability of romantic love: A reliability generalization meta-analysis. Personal Relationships, 16(1), 49-66. doi: http://dx.doi. org/10.1111/j.1475-6811.2009.01209.x.

Hendrick, C. \& Hendrick, S. (1986). A theory and method of love. Journal of Personality and Social Psychology, 50(2), 392-402. doi: http://dx.doi.org/10.1037/00223514.50.2.392.

Hendrick, C. \& Hendrick, S., (1989). Research on Love: Does It Measure Up? Journal of Personality and Social Psychology 56 (5), 784-794. doi: http://dx.doi.org/1989-24552001.

Hendrick, S., Hendrick, C., \& Dicke, A. (1998). The Love Attitude Scale: Short Form. Journal of Social and Personal Relationships, 15(2), 147-159. doi: http://dx.doi. org/10.1177/0265407598152001.

Jonason, P. K. \& Kavanagh, P. (2010). The dark side of love: The Dark Triad and love styles. Personality and Individual Differences, 49(6), 606-610. doi: http://dx.doi.org/10.1016/j. paid.2010.05.030.

Kline, P. (1986). A Handbook of Test construction: Introduction to psychometric design. London: Methuen.

Lee, J. A. (1973). The colours of love: An exploration of the ways of loving. Toronto: New Press.

Lema, L., Salazar, I., Valera, M., Tamayo, J., Rubio, A., \& Botero, A. (2009). Comportamiento y salud de los jóvenes universitarios: Satisfacción con el estilo de vida. Pensamiento psicológico, 5(12), 71-88. Recuperado de http://portales. puj.edu.co/psicorevista/components/com_joomlib/ebooks/ PS12-5.pdf.

Lloret-Segura, S., Ferreres-Traver, A., Hernández-Baeza, A., \& Tomás-Marco, I. (2014). El análisis factorial exploratorio de los ítems: Una guía práctica, revisada y actualizada. Anales de Psicología, 30(3), 1151-1169. doi: http://dx.doi. org/10.6018/analesps.30.3.199361.

Mardia, K. V. (1970). Measures of multivariate skewnees and kurtosis with applications. Biometrika, 57(3), 519-530. doi: http://dx.doi.org/10.2307/2334770.

Muñiz, J., Elosua, P., \& Hambleton, R. K. (2013). Directrices para la traducción y adaptación de los tests: segunda edición. Psicothema, 25(2), 151-157. doi: http://dx.doi. org/10.7334/psicothema2013.24. 
Neto, F. (2012). Compassionate Love for a Romantic Partner, Love Styles and Subjective Well-Being. Interpersona: An International Journal on Personal Relationships, 6(1), 2339. doi: http://dx.doi.org/10.5964/ijpr.v6i1.88.

Osborne, J. (2014). Best Practices in Exploratory Factor Analysis. Recuperado de https://www.researchgate.net/ publication/265248967_Best_Practices_in_Exploratory_Factor_Analysis.

Otazzi, A. (2009). Estilos de amor, satisfacción, y compromiso en relaciones de pareja estables. (Tesis de Licenciatura, Pontificia Universidad Católica del Perú). Recuperado de http:// tesis.pucp.edu.pe/repositorio/handle/123456789/430.

Prieto, G. \& Delgado, A. (2010). Fiabilidad y validez. Papeles del Psicólogo, 31(1), 67-74. Recuperado de http:/www.papelesdelpsicologo.es/resumen?pii=1797.

Prieto, L., Lamarca, R., \& Casado, A. (1998). La evaluación de la fiabilidad en las observaciones clínicas: el coeficiente de correlación intraclase. Medicina Clínica, 110, 142-145. Recuperado de http://www.elsevier.es/es-revista-medicinaclinica-2-articulo-la-evaluacion-fiabilidad-las-observaciones-2202.

Rodríguez, I. A., Montgomery, M., Peláez, M., \& Salas, W. (2003). Actitudes amorosas y experiencias en el cortejo de adultos y jóvenes de tres distintas culturas. Revista Mexicana de Psicología, 20(2), 177-188. Recuperado de http:// www2.fiu.edu/ pelaeznm/pdfs/14.Love $\% 20$ attitudes $\% 20$ and $\% 20$ dating $\% 20$ experiences.pdf.

Rodríguez-Castro, Y., Lameiras, M., Carrera, M. V., \& Vallejo-Medina. P. (2013). Validación de la Escala de Actitudes hacia el Amor en una muestra de adolescentes. Estudios de Psicología, 34(2), 209-219. doi: http://dx.doi. org/10.1174/021093913806751429.

Sánchez-Meca, J. \& López-Pina, J. (2008). El enfoque metaanalítico de generalización de la fiabilidad. Acción Psicológica, 5(2), 37-64. Recuperado de http://www.um.es/ metaanalysis/pdf/5020.pdf.

Serra-Mayoral, A. \& Peña-Casanova, J. (2006). Fiabilidad test-retest e interevaluador del Test Barcelona. Neurología, 21(6), 277-281. Recuperado de http://publicfiles.prbb.org/publicacions/02559081-11db-4227-b3c94b447c3f6527.pdf.

Tabachnick, B. \& Fidell, L. (2013). Using multivariate statistics (6th.ed.). Nueva Jersey: Pearson.

Thompson, E. R. (2007). Development and Validation of an Internationally Reliable Short-Form of the Positive and Negative Affect Schedule (Panas). Journal of CrossCultural Psychology, 38(2), 227-242. doi: http://dx.doi. org/10.1177/0022022106297301.
Timmerman, M. E. \& Lorenzo-Seva, U. (2011). Dimensionality assessment of ordered polytomous items with parallel analysis. Psychological Methods, 16(2), 209-220. doi: 10.1037/a0023353.

Todosijević, B., Arančić, A., \& Ljubinković, S. (2009). An Examination and Revision of the Love Attitude Scale in Serbia. Interpersona: An International Journal on Personal Relationships, 3(1), 58-76. doi: https://doi.org/10.5964/ijpr. v3i1.32.

Ubillos, S., Páez, D., \& Zubieta, E. (2004). Relaciones íntimas: atracción amor y cultura. En D. Páez, I. Fernández, S. Ubillos \& E. Zubieta (Eds.), Psicología social, cultura y educación (pp. 511-536). Madrid: Pearson Prentice Hall.

Ubillos, S., Zubieta, E., Páez, D., Deschamps, J. C., Ezeiza, A., \& Vera, A. (2002). Amor, Cultura y Sexo. Revista Española de Motivación y Emoción, 3, 197-221. Recuperado de http://reme.uji.es/articulos/aubils9251701102/texto.html.

Valdéz, J. L., González, N. I., \& Sánchez, Z. P. (2005). Elección de pareja en universitarios mexicanos. Enseñanza e Investigación en Psicología, 10(2), 355-367. Recuperado de http:// www.redalyc.org/articulo.oa?id=29210210.

Velicer, W. F. \& Fava, J. L. (1998). Effects of variable and subject sampling on factor pattern recovery. Psychological Methods, 3(2), 231-251. doi: http://dx.doi. org/10.1037/1082-989X.3.2.231.

Wan, W. S., Hoesni, S. M., \& Chong, S. T. (2012). Investigating the Factor Structure of the Love Attitude Scale (LAS) with Malaysian Samples. Asian Social Science, 8(9), 66-73. doi: http://dx.doi.org/10.5539/ass.v8n9p66.

Watson, D., Clark, L. A., \& Tellegen, A. (1988). Development and validation of brief measures of positive and negative affect: The Panas scales. Journal of Personality and Social Psychology, 54(6), 1063-1070. doi: 10.1037//0022-3514.54.6.1063.

White, J. K. (2003). The five-factor personality variables and relationship variables: A study of associations. (Unpublished doctoral dissertation, Texas Tech University). Recuperado de https://tu-ir.tdl.org/ttu-ir/bitstream/ handle/2346/10580/31295018529841.pdf? sequence=1

Zwick, W. \& Velicer, W. (1986). Comparison of five rules for determining the number of components to retain. Psychological Bulletin, 99(3), 432-442. doi: http:// dx.doi.org/10.1037/0033-2909.99.3.432. 